\title{
Intralobar pulmonary sequestration of ectopic pancreatic tissue with gastropancreatic duplication
}

\author{
B CORRIN, C DANEL, A ALLAWAY, JO WARNER, W LENNEY \\ From the Brompton Hospital, London, and the Royal Alexandra Hospital for Sick Children, Brighton
}

Pancreatic tissue has seldom been described in the lung. In a recent patient it was an unexpected postmortem finding, but in retrospect the clinical findings correlated well with the presence of ectopic pancreatic tissue in the lung.

\section{Case report}

A white baby girl weighing $3120 \mathrm{~g}$ was born at term by normal vaginal delivery. Her 35 year old mother had previously suffered 12 years of primary infertility and this pregnancy had been complicated by essential hypertension and polyhydramnios. The Apgar scores were 9 and 10 at 1 and 5 minutes respectively. Routine examination showed an imperforate anus and an enlarged right labium majus. At 1 hour of age she developed mild grunting and tachypnoea; she was given $30 \%$ oxygen and transferred to the Royal Alexandra Hospital for Sick Children, Brighton. The respiratory symptoms settled quickly. She passed meconium per vaginam and her rectovaginal fistula was dilated to size 6 Hagar. She was not eager to feed and as there was some vomiting she was given intravenous fluids for the first 48 hours. Daily dilatations of the fistula were continued over the first week of life, increasing it gradually to size 12 Hagar. Nasogastric milk feeds commenced on day 3 and provided most of her fluid requirements by day 7. On day 8 she developed a persistent fever, tachypnoea, crepitations at the right lung base, and a distended abdomen. Chest radiographs showed collapse and consolidation of the right lower lobe. Gastric feeds were stopped, intravenous penicillin and gentamicin were started, and within 48 hours she was much improved. Coliform bacteria sensitive to gentamicin were grown from her blood. Milk feeds were restarted on day 11 but by day 13 she had deteriorated with recurrent fever, tachypnoea, and crepitations at the right lung base. Chest radiographs showed right lower lobe consolidation as previously, together with a fluid filled cavity. The antibiotic regimen was changed to intravenous cefotaxime, intravenous feeding was restarted, oral milk feeds were stopped, and once more there was improvement within 48 hours. Over the next 10 days two attempts were made to restart milk feeds but within 24 hours of each the respiratory rate increased and the baby looked less well. On day 24 dark, altered blood was aspirated through the nasogastric tube and two large melaena stools were passed. The haemoglobin concentration fell to $8.9 \mathrm{~g} / \mathrm{dl}$ and she required a transfusion of $80 \mathrm{ml}$ fresh

Address for reprint requests: Professor B Corrin, Cardiothoracic Institute, Brompton Hospital, London SW3 6HP.

Accepted 15 March 1985 blood. The results of clotting studies and urea and electrolyte concentrations were normal. A fistula between the oesophagus and the right lung was suspected but a barium swallow and technetium milk feed scan were normal. A barium meal showed a constant rounded filling defect in the greater curvature of the stomach, suggesting a duplication cyst; but there was no communication with normal bowel. Repeat chest radiography showed further deterioration, with several fluid filled cysts in the right lower zone. On day 28 the baby was transferred to the Brompton Hospital. Her condition continued to worsen, pseudomonas was grown from pharynx and blood, and despite appropriate intravenous antibiotic treatment she died aged 31 days.

Necropsy confirmed the imperforate anus and rectovaginal fistula. Also found was a gastric duplication cyst, $1.5 \mathrm{~cm}$ in diameter, and a strip of pancreatic tissue that duplicated the normal pancreas. Neither duplication communicated with the duodenum. A cord of fibrous tissue connected the gastric and pancreatic duplications to the lower lobe of the right lung, accompanied through the right side of the diaphragm by an aberrant muscular artery from the coeliac axis (fig 1). Microscopy showed that this lacked a duct or any other epithelial component. Both lungs were very congested and firm, with necrotic foci evident through the visceral pleura. Slicing the lungs showed a fairly well circumscribed but non-encapsulated multicystic lesion within the right lower lobe. The cysts were lined by columnar, pseudostratified ciliated epithelium but did not communicate with the airways and lacked cartilage in their walls. Microscopy also showed ectopic pancreatic tissue (fig 2) and foci of necrosis intimately related to the cysts. Islets of Langerhans were not readily identified in haematoxylin and eosin stained sections but immunocytochemical examination showed foci of insulin and glucagon containing cells. Arteries related to the necrotic lung tissue were colonised by numerous bacilli. The right upper and middle lobes also showed arterial colonisation by bacilli and patchy necrosis. The left lung showed proteinaceous intra-alveolar exudates and non-specific interstitial pneumonia, worse in the lower lobe. The appearances in all lobes of the right lung were those of pseudomonas pneumonia while the lower lobe was also the site of ectopic pancreatic tissue.

\section{Discussion}

By the time our patient died there was a well established pseudomonas pneumonia, which with the characteristic colonisation and necrosis of arterial walls and subsequent tissue ischaemia could well account for much of the lung 


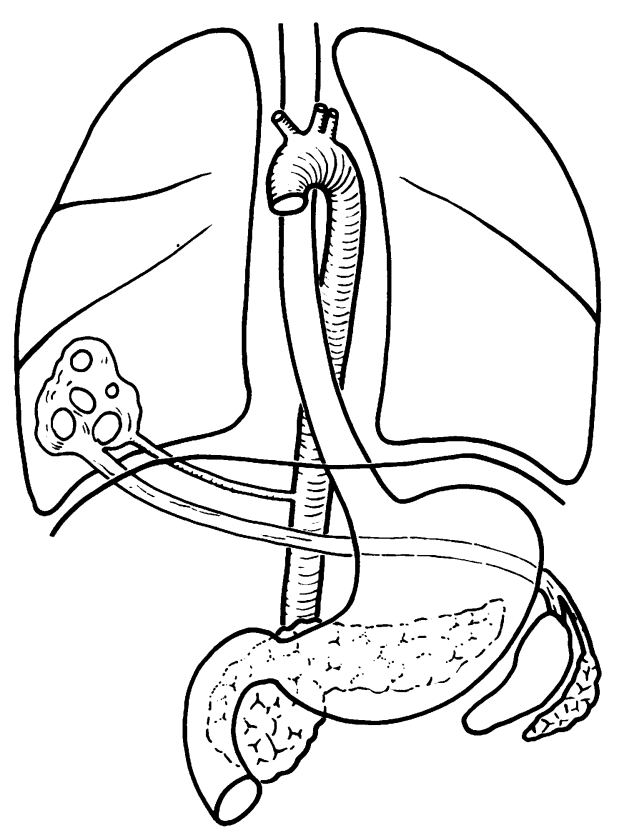

Fig 1 The abnormalities found at necropsy.

necrosis evident at necropsy. Our clinical and radiological observations in life, however, indicate severe pulmonary problems directly related to feeding well before this terminal pneumonia, and enzymatic digestion of the lung due to the presence of ectopic pancreatic tissue provides a more attractive explanation for the episodic respiratory distress.

The pulmonary cysts were lined by respiratory epithelium, yet lacked cartilage in their walls, and in this respect had the structure of undifferentiated foregut cysts. The presence of pancreatic tissue within their walls, however, categorises them as enteric. Their connection to a gastropancreatic duplication by a fibrous band passing through the diaphragm suggests that the whole anomaly arose as an enteric duplication. Two comparable cases have been reported previously. Beskin ${ }^{1}$ described a congenitally cystic left lower lobe containing ectopic pancreatic tissue, supplied by an anomalous systemic artery and connected to the gastro-oesophageal junction by an oesophageal duplication; he termed this lesion an intralobar enteric sequestration. There is a close similarity to our case and to case 3 of Baar and d' Abreu, ${ }^{2}$ in which ectopic pancreatic tissue connected an oesophageal diverticulum to the lungs. The fourth case of Baar and d' Abreu is also of interest: here an intrapericardial bronchoenteric cyst contained ectopic pancreas in its walls. Collectively these cases link duplication of the foregut with enteric cysts; accessory lungs and pulmonary sequestrations probably represent further varieties of foregut duplications. ${ }^{34}$

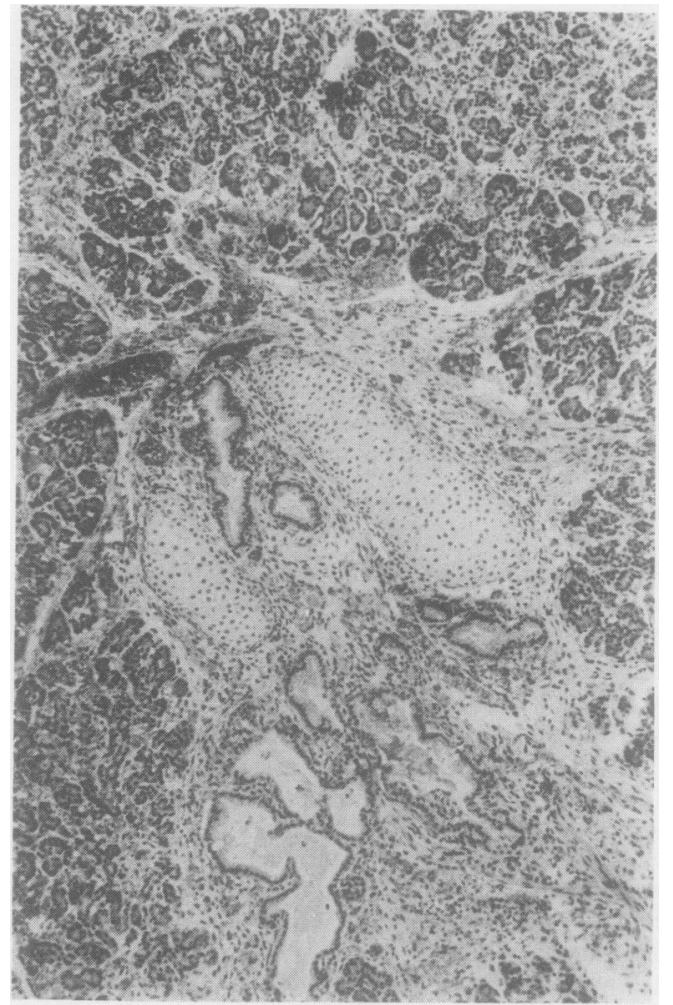

Fig 2 Part of the right lower lobe showing a bronchus surrounded by pancreatic acini. (Haematoxylin and eosin, $\times 55$.)

We are indebted to $\operatorname{Dr} E$ Heyderman for the immunocytochemical demonstration of insulin and glucagon.

\section{References}

1 Beskin CA. Intralobar enteric sequestration of the lung containing aberrant pancreas. J Thorac Cardiovasc Surg 1961;41:314-7.

2 Baar HS, d Abreu AL. Duplication of the foregut. Superior accessory lung ( 2 cases); epiphrenic oesophageal diverticulum; intrapericardial teratoid tumour; and oesophageal cyst. $\mathrm{Br} J$ Surg 1949;37:220-30.

3 Flye MD, Izant RJ. Extralobar pulmonary sequestration with esophageal communication and complete duplication of the colon. Surgery 1972;71:744-52.

4 Heithoff KB, Sane SM, Williams HJ, et al. Bronchopulmonary foregut malformations: a unifying etiological concept. Am J Roentgenol 1976;126:46-55. 